\title{
Planned Active Spectroscopy in the Neutral Beam Injectors of W7-X
}

\author{
Juergen Baldzuhn ${ }^{1}$, Oliver Ford, Paul McNeely, Torsten Richert, W7-X Team \\ Max-Planck Institut fuer Plasmaphysik, EURATOM Assoc., \\ 17491 Greifswald, Germany
}

\begin{abstract}
The new stellarator Wendelstein 7-X will employ a combination of beam-aided active spectroscopy systems. Active Charge Exchange Resonance Spectroscopy will be performed in the beam of a diagnostic neutral beam injector. This low power, low divergence neutral beam system allows for the spectroscopic measurement of impurity density profiles, ion temperature and radial electric field profiles with good spatial resolution. However, no toroidal rotation can be measured with it, because of the radial arrangement of the observation chords. This instead will be done by CXRS in the neutral NBI heating beams, in addition to Beam Emission Spectroscopy to monitor the beam absorption in the plasma. Finally, the Motional Stark Effect diagnostics in the NBI can measure the magnetic pitch angle to provide information on iota variations by the bootstrap current. This information is essential for reliable W7-X divertor performance, which depends strongly on the edge iota value. In this paper the beam injectors are described together with the beam properties and observation geometries. Details are given for the spectroscopic systems. The relevance of the observed plasma parameters is briefly discussed, with an emphasis on some stellarator optimization criteria.
\end{abstract}

KEYWORDS: Plasma diagnostics - spectroscopy

First EPs Conference on Plasma Diagnostics - $1^{\text {st }}$ ECPD

14-17 April 2015,

Villa Mondragone, Frascati (Rome) Italy

\footnotetext{
${ }^{1}$ Speaker: baldzuhn@ipp.mpg.de 


\section{Introduction}

The stellarator Wendelstein 7-X (W7-X) [1] is a fully optimized plasma fusion experiment. It utilizes a system of 70 superconducting coils [2] to confine the plasma in a toroidal magnetic field with a magnetic flux density of up to $3 \mathrm{~T}$ in the plasma centre. To maintain the required plasma temperature of several $\mathrm{keV}$, microwave heating by Electron Cyclotron (ECRH), Ion Cyclotron Resonance Heating (ICRH) and Neutral Beam Injection (NBI) heating will be used. For nuclear fusion, one of the most essential plasma parameters is the ion temperature $T_{i}$, which

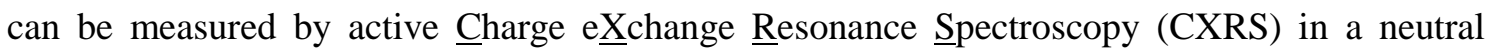
beam. For that purpose, besides the heating NBI an additional RUssian Diagnostic neutral beam Injector on W7-X (RuDI-X) [3] will be installed. In addition to the CXRS system, a Neutral Particle Analyzer system (NPA) will be used. It will measure the energy spectra of the neutral particles leaving the plasma volume after charge exchange with the neutrals of the beam. Essential for the stellarator plasma confinement is the radial electric field $E_{r}$ which is measured by evaluation of the spectral line Doppler shift of the CXRS impurity line. Beam Emission Spectroscopy (BES) and Motional $\underline{S}$ tark Effect (MSE) techniques allow for the evaluation of the magnetic pitch angle in the plasma by observation of the hydrogen Balmer-alpha Stark multiplet pattern. A combination of MSE spectroscopy, polarimetry and coherence Imaging techniques [4] (IMSE) are foreseen. Fig. 1 shows the arrangement of the W7-X plasma vessel together with the heating ports and diagnostics ports used by the active spectroscopy methods.

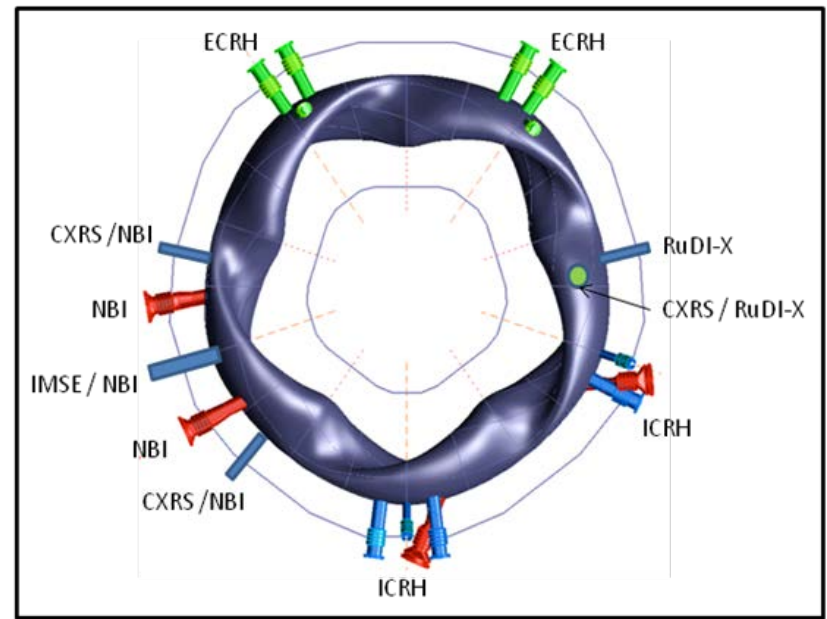

Fig. 1: Sketch of the W7-X plasma vessel (top-view) together with the heating ports for the ECRH, the NBI, the ICRH, for RUDI-X and the diagnostic ports for the CXRS and IMSE.

\section{The neutral beam injectors}

In this chapter, we describe briefly W7-X's neutral beam injectors. The diagnostic neutral beam injector RuDI-X had been developed and built by the Budker Institute in Novosibirsk, Russia. It provides a low divergence (half angle $<0.7^{\circ}$ ) neutral beam with a high fraction of neutrals in the full energy component (>50\%). It has a total equivalent neutral current $>5 \mathrm{~A}$ in order to optimize the CXRS light output. The acceleration voltage is variable from $20 \mathrm{kV}$ to 60 $\mathrm{kV}$. A narrow beam waist $(\Phi<15 \mathrm{~cm})$ combined with the low divergence provides a good spatial resolution for the CXRS measurements in the plasma. The variable acceleration voltage allows tuning to maximize the CX light emission efficiency for the impurity species under consideration. This can be helium, boron, carbon, nitrogen, oxygen or neon. The relatively low beam power $(<300 \mathrm{~kW})$ compared to the heating beams allows for measurements during pure 
ECRH discharges without excessive particle and power feed. The neutral beam will be modulated at $10-100 \mathrm{~Hz}$ to allow for background subtraction of the plasma radiation. The beam axis is oriented perpendicularly to the magnetic axis to improve the spatial CXRS resolution and to provide the poloidal component of the $E X B$ drift velocity vector resulting from $\mathrm{E}_{\mathrm{r}}$. The current four-grid design allows at maximum 5 second pulses. The ion source employs a rfexcited hydrogen plasma, using a $20 \mathrm{~kW}, 4-5 \mathrm{MHz}$ generator. The RuDI-X vacuum tank is pumped by two Leybold cryo-pumps with $60.000 \mathrm{l} / \mathrm{s}$ and $30.000 \mathrm{l} / \mathrm{s}$ pumping speed. The first pump is used to pump the ion source, the beam neutralizer and the ion deflector. The second pump is for the beam duct towards the W7-X vessel to avoid beam blocking in case of a too high power deposition inside this duct. The special charcoal coating of the cryo-panels optimizes the pumping speed for highly energetic particles.

Two NBI injector boxes [5] are installed with radial orientation on W7-X. Due to the almost perpendicular injection relative to the magnetic field the toroidal momentum input is negligible. Each injector box can hold 4 ion sources, each mounted on a Plug In Neutral Injector (PINI). At the start of the W7-X operation, only 2 PINIs per box will be installed. The injector box also contains: the neutralizers, the ion deflector magnet, the ion dumps and the high speed vacuum pumps to evacuate the neutral gas. High speed pumping during beam extraction is accomplished by means of titanium getter pumps with ac-current heated filaments to allow operation in the permanent magnetic field of W7-X. For hydrogen operation, each PINI provides a nominal heating power of $1.7 \mathrm{MW}$ at $55 \mathrm{kV}$ extraction voltage. In deuterium operation the average heating power increases to $2.3 \mathrm{MW}$ at $60 \mathrm{kV}$ extraction voltage. The maximum pulse length is limited to $10 \mathrm{~s}$. In the future, longer NBI plasma phases can therefore be accomplished only by sequential operation of the individual PINIs.

\section{The spectroscopic systems}

Some aspects of the active spectroscopy on W7-X are described in this chapter. Fig. 2 shows a poloidal cross section of W7-X, together with the RuDI-X injection geometry and the observation chords for the CXRS and NPA diagnostics. In the neutral beam of RuDI-X, only one CXRS system will be available during the first operational phase of W7-X. A fan of 10 observation chords will be installed in a water cooled immersion tube with a sapphire window because of the mechanical stability compared to quartz. The immersion tube, window and mechanical shutter are fully compatible to high-power, long-pulse discharges (30 mins) from the beginning on. The window is coated with a layer of $1,4 \mu \mathrm{m}$ thick ITO (Indium Tin $\underline{\text { Oxide) with }}$ high electric conductivity $(<10 \Omega$ /square) to absorb the ECRH stray radiation inside the torus. The CXRS light is imaged to the 10 bundles of quartz fibers, which transmit the light to two Czerny-Turner spectrometers with 5 bundles (50 fibres per bundle) each. The light from the bundles is transferred into the spectrometers via an intermediate imaging optics to match the étendue. For the central plasma channels, a spectrometer focal length of $500 \mathrm{~mm}$ is used, for the edge channels $750 \mathrm{~mm}$. High-sensitivity CCD cameras are installed in the exit plane of the spectrometers. The use of that type of spectrometers provides a large flexibility with respect to the choice of the impurity species. However, because of the poloidal orientation of the observation chords, only the poloidal component of the plasma rotation is perceptible. Major tasks for this arrangement will therefore be the measurement of $\mathrm{T}_{\mathrm{i}}, \mathrm{E}_{\mathrm{r}}$ and impurity density profiles. Because of the high light-feedthrough, also time resolved measurements should be possible with a maximum time resolution of less than $10 \mathrm{~ms}$. The active CXRS system will be complemented by a passive spectroscopic system with also 10 observation chords, situated close 
to the plasma edge at another toroidal location. For the passive system only spectral lines from electron impact excitation will be observable. It will provide line integrated edge values of $T_{i}$ and $E_{r}$. Five radial chords for the NPA system are planned, each of them providing an entire particle spectrum with 10 energy channels.

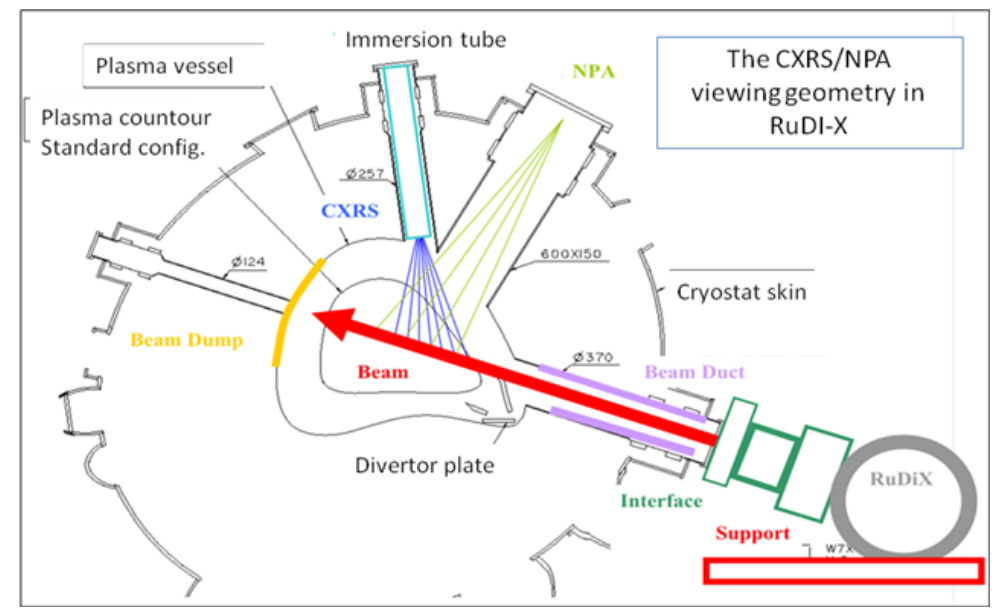

Fig. 2: Poloidal cross section with the RuDI-X injection geometry and the CXRS and NPA observation chords.

For the observation of the heating NBI beams three ports are available (see fig. 1); all lying almost in the equatorial plane of W7-X. The port lying between the NBI-ports will be used for the IMSE system. In contrast to the spectroscopy of the individually polarized multiplet components, the imaging technique utilizes $2 \mathrm{D}$ information. This has the major advantage of a high useful MSE light intensity, while the full polarization information is used for the reconstruction of the iota-profile. However, in contrast to tokamaks, the net toroidal currents in W7-X are small, making a thorough numerical modelling in advance of the measurement mandatory. The ports left and right of the NBI-ports will be used for CXRS and BES. In all observation ports, water cooled immersion tubes will be used with cooled shutters in front of the windows. The optical concept and technical set-up for CXRS and BES in the heating beams will be comparable to the CXRS system of the RuDI-X. However, because of the almost toroidal orientation of the observation chords, no $E_{\mathrm{r}}$ measurement will be possible. The ExB-drift in W7$\mathrm{X}$ will be mainly into the poloidal direction. Another drawback is the fact that the diameter of the heating beams is comparable to the radial extension of the plasma itself, reducing the number of useful radial channels in the plasma. A maximum of 5 radial channels are possible and will be realized. The BES will measure the NBI beam absorption in the plasma, i.e. the NBI power and particle deposition profile.

\section{The numerical simulations}

A MATLAB code [6] was written to simulate the expected CXRS, NPA and BES spectra. This code calculates the beam absorption for RuDI-X and the NBI as a function of the plasma parameters, and uses the ADAS atomic data [7] to predict the spectral line shape and line intensity for the CXRS lines and BES lines for a variety of impurity species. The magnetic field configuration is taken from VMEC equilibria [8]. In addition, the poloidal plasma rotation due to $E_{\mathrm{r}}$ is also taken into account. Background light emission from the "cold" plasma edge after electron impact excitation is calculated to investigate the impact of perturbing impurity spectral 
line contributions to the CXRS and BES signals. STRAHL code [9] impurity transport calculations are performed to match the edge spectral lines (the impurity particle sources) to the central impurity density (CXRS light) profiles. The radial profiles for diffusion and convection are chosen such that neoclassical or anomalous impurity transport is modelled. Because no W7$\mathrm{X}$ impurity transport code is available, so far, a comparable tokamak plasma is used for that choice. All optical parameters of the quartz fibres and spectrometers are included in the MATLAB code, artificial noise can be added to the simulated spectra. The performance of the future CCD cameras can freely be chosen. The beam and observation chord geometry is taken from CATIA [10] models which were used to build the hardware. A heuristic slowing-down model for the fast beam particles is used to simulate the NPA spectra. In addition, a suprathermal population of fast impurity ions can be included (for instance for helium ions after injection by neutral beam injectors or during ICRH discharges) to investigate the possibility of CXRS on fast ion populations.

The major objective of this code is the prediction of the quality of spectroscopically measured plasma parameters as a function of the beam and plasma parameters during the future W7-X operation. The influence of noise can be assessed, operational margins or limits for the acceleration voltage, plasma density etc. can be evaluated in advance. The error bars of the measured plasma parameters can be estimated, and experimental conditions can be anticipated as a function of the desired experimental outcome. Fig. 3 shows calculated examples of a motional Stark pattern and a CXRS spectrum in the RuDI-X beam.

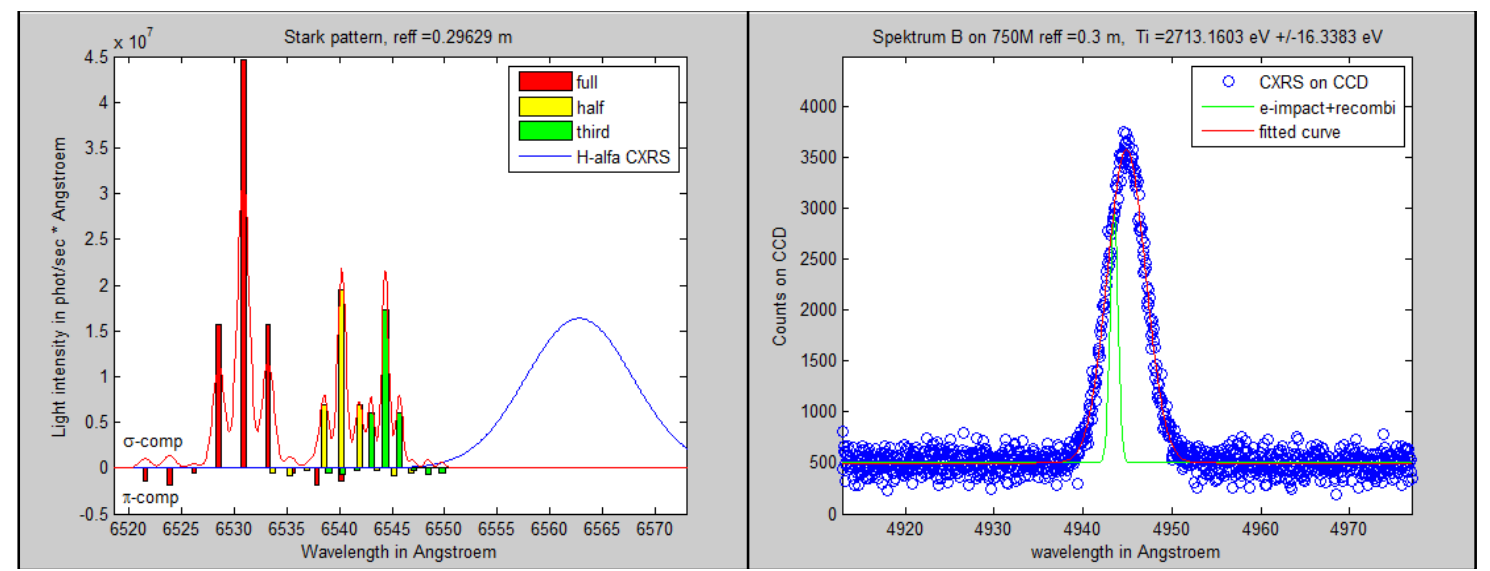

Fig. 3: Left side: calculated spectra for the MSE excited pattern of the active Balmer-alpha emission with all $\sigma$-and $\pi$-components for the three energetic beam contributions, the active CRXS hydrogen line is shown as solid blue line. Right side: a spectrum of the CXRS excited line of Boron-V including noise (blue circles), the fit to a Gaussian (red line) and the electron impact excited spectral line contribution from the plasma edge (green line).

\section{Some aspects of the optimization}

In this chapter, briefly some aspects of the stellarator optimization [11] will be discussed, with emphasis on the mentioned active spectroscopy diagnostics. The most important task of W7-X will be the demonstration of the optimization criteria for a stellarator with regard to a future fusion reactor. One of these criteria is the confinement of high energetic particles according to the quasi-isodynamic approach [12], in this case the good confinement of the fusion alphas, until they slowed down sufficiently to deposit their energy in the plasma. After that, the helium ash should be removed as quickly as possible. CXRS provides He density profiles to 
assist the corresponding investigations, not only for helium but also for several other light impurities. Besides helium transport, CXRS can; therefore, also deliver information on the impurity transport for discharge conditions that might be subject to impurity accumulation. Strong negative $E_{\mathrm{r}}$ in the ion-root might enhance this accumulation. The numerical simulations predict; however, large problems in resolving fast ion population by CXRS directly, for instance after beam injection of helium or during ICRH heating. Another optimization criterion, the reduction of the intrinsic neoclassical transport properties down to a comparable tokamak level, can be sustained with the support of $E_{r}$, either by a direct reduction of the neoclassical transport coefficients, or by the formation of a shear layer with the subsequent suppression of instabilities, i.e. the anomalous transport. CXRS will deliver the radial profile of $E_{r}$ by the measurement of the poloidal plasma rotation. Of particular interest is the formation of positive $E_{r}$, the electron-root, because this might help to expel efficiently all impurities. The numerial simulations (see above) predict the possibility of using time resolved CXRS measurements to measure the impurity density by beam modulation, for instance, after impurity gas puff or laser ablation/ impurity pellet injection. The next optimization criterion is the reduction of the parallel currents, mainly the Pfirsch-Schlüter and bootstrap-current. Strong parallel currents will provide a strong Shafranov shift for high plasma beta, an effect which is minimized in W7-X. Nevertheless, several $10 \mathrm{kA}$ of bootstrap current are predicted for W7-X, which will influence the iota-profile. The actual value of the edge iota(a), however, is essential for the performance of the open island divertor concept. The positioning of the divertor strike lines with high power deposition is decisive for the divertor pumping efficiency and the impurity release by sputtering and erosion. MSE provides the means to measure directly the magnetic pitch angle, i.e. the iota-profile. BES in the neutral beams will provide direct information on the NBI power deposition in the plasma, a crucial input for all energy transport calculations. This will help to evaluate whether a neoclassical transport level is maintained in $\mathrm{W} 7-\mathrm{X}$, as predicted for the optimized magnetic configuration, and in how far anomalous transport plays a role.

\section{References:}

[1] G. Grieger, et al. Physics optimization of stellarators, Phys. Fluids B 4 (1992), p. 2081-91.

[2] J. Sapper, Superconducting coil development for the stellarator W7-X, Fusion Engineering and Design 20 (1993), p. 23-32.

[3] T. Richert et al. Diagnostic neutral beam injector for W7-X, 25th Symposium on Fusion Engineering (SOFE 2013), ID: 667715.0

[4] J. Howard et al. Snapshot-imaging motional Stark effect polarimetry, Plasma Phys. Contr. Fusion 50 (2008), 125003 (18pp).

[5] P. McNeely et al. Current status of the neutral beam heating system of W7-X, Fusion Eng. and Design 88 (2013) p. 1034.

[6] The Mathworks Inc, www.mathworks.com

[7] Open-ADAS: www.adas.ac.uk

[8] S. P. Hirshman, et al. Steepest-descent moment method for three-dimensional magnetohydrodynamic equilibria, Phys. Fluids 26 (1983) p. 3553.

[9] K. Behringer, Description of the impurity code STRAHL, Plasma Phys. and Fusion Tech. 19 (1987), JET-R-87-08.

[10] CATIA- Dassault systems, www.3ds.com

[11] R. Wolf, A stellarator reactor based on the optimization criteria of Wendelstein 7-X, Fusion Eng. and Design 83 (2008), p. 990-996.

[12] J. Nührenberg, Plasma Phys. Control. Fusion 52 (2010), 124003 